\title{
Effects of sentence writing in second language lexical acquisition
}

\author{
Joe Barcroft Washington University
}

This study compared the effects of writing new words in sentences with word-picture repetition learning alone. Second language (L2) Spanish learners attempted to learn 24 new Spanish words in one of two conditions while viewing word-picture pairs. In Experiment 1, in the no sentence writing condition, the participants viewed 4 repetitions of each word for 6 seconds each. In the sentence writing condition, they viewed 1 repetition of each word for 48 seconds and were asked to write the word in a Spanish sentence. In Experiment 2, the participants were shown one repetition of each word for 24 seconds in both the sentence writing and no sentence writing conditions. Immediate and delayed posttests on productive vocabulary knowledge were administered in both experiments. Scores were submitted to analyses of variance. Condition and time were independent variables. Target word production was scored based on syllables and whole words produced. Results of both experiments indicated strong negative effects for the sentence writing conditions, suggesting that sentence writing can inhibit word form learning during the initial stages of L2 lexical acquisition.

\section{Introduction}

In recent years, second language (L2) researchers have become increasingly interested in the central role of lexical acquisition in language acquisition and in the role of acquired lexical knowledge in the acquisition of syntax. To a large degree, improving our understanding of L2 acquisition depends on improving our understanding of how learners acquire individual words and word parts. Although the quantity of empirical data on incidental and direct 
L2 vocabulary acquisition has increased substantially within the past two decades, more cognitively oriented research programs are needed to address key issues in this area. One cognitively oriented branch of L2 vocabulary research developed in recent years has focused on word-level input processing, or how learners process the various subcomponents of L2 words (formal, semantic, mapping) when these words appear in the input. The present study, oriented within this line of research, examined the effects of requiring learners to write target L2 words in original sentences during the initial stages of L2 word learning. By comparing the effects of these conditions, the study explored the relationship between subtasks associated with sentence writing, such as semantic elaboration and output, and L2 word learning.

Writing a new word in a sentence involves both semantic elaboration and output. It involves semantic elaboration because a learner must retrieve the meaning of a word and the contexts in which it can be used to a sufficient degree in order to be able to write the word in a sentence. It involves output because sentence writing by its nature requires production on the part of the learner. Some researchers have posited that the type of semantic elaboration involved in sentence writing should facilitate learning new words (e.g., target pseudowords; Coomber et al., 1986). Other researchers have argued that the type of output involved in sentence writing can facilitate lexical learning (Laufer, 1997). However, there is no immediate reason to believe that either semantic elaboration or output should positively affect L2 lexical learning, especially with regard to the formal properties of new words, because both semantic elaboration and output utilize processing resources that could otherwise be directed towards encoding and retaining new word form. The review of lexical studies on semantic elaboration, output and vocabulary learning in the following section leads up to and supports this assertion.

\section{Semantic elaboration and memory for words}

Semantic elaboration refers to a situation in which a learner's processing resources are directed at the semantic (referential, meaning-related) properties of a stimulus item in the input, that is, 
a situation in which a learner is focused on semantic properties than would otherwise be the case. Two examples of items that can be semantically elaborated upon would be (1) known words that one has already acquired and (2) new words that one has not yet acquired but for which one has access to their meaning (e.g., viewing a new word along with a picture of that word). An example of a task that would require semantic elaboration on known words would be to judge whether a known word in one's first language (L1) represents an instance of a particular concept (e.g., 'economic') as opposed to crossing out vowels in the word or copying the word (Tresselt and Mayzner, 1960). On the other hand, an example of a task that would require semantic elaboration on new words would be to judge whether a new word represents an instance of a particular concept (e.g., 'economic') as opposed to crossing out vowels in the word or copying the word while one also has access to the meaning of the word (e.g., via pictures, translation or context). Writing a new word in a sentence also involves semantic elaboration because, in order to do so, a learner must evaluate semantic properties of the word so as to be able to place the word in some appropriate sentence-level context.

Previous studies have found that semantic elaboration positively affects memory for known words (Tresselt and Mayzner, 1960; Hyde and Jenkins, 1969; Bower and Reitman, 1972; Schulman, 1974; Craik and Tulving, 1975; Epstein et al., 1975; Johnson-Laird et al., 1978; Ross, 1981) and memory for other types of stimuli (e.g., L1 sentence recall: Stevenson, 1981; L1 text recall: McDaniel, 1984). Such findings are in line with the levels of processing (LOP) theory of human memory (Craik and Lockhart, 1972), which posits that memory for an item depends on the level or relative depth at which the item is processed in a learner's cognitive system. Activities requiring more elaborate manipulation of information (deeper processing) result in better memory than their counterparts (shallower processing).

The LOP framework has inspired the development of imagerybased mnemonic vocabulary learning techniques (for a review, see Cohen, 1987), such as Keyword (Atkinson and Raugh, 1975), and some research on semantic elaboration and lexical acquisition 
that does not involve the use of mnemonics. From a pedagogical standpoint, the LOP framework has also been interpreted as support for the idea that semantic elaboration should lead to more effective vocabulary learning. For example, Johnson and Pearson (1978; 1984) proposed 'semantic features analysis' and 'semantic mapping' as effective vocabulary instruction techniques (on semantic elaboration and vocabulary learning, see also Stahl and Fairbanks, 1986; Brown and Perry, 1991). Many language instructors also maintain that semantic elaboration ('focus on word meaning') should facilitate vocabulary learning, in other words, that it should be useful for students to engage in semantically elaborative activities when attempting to learn new words (e.g., talking about word meanings, relating new words to personal experiences, focusing on usage contexts, comparing and contrasting new words, writing new words in sentences).

The limited number of studies on semantic elaboration and new word learning (apart from mnemonic techniques such as Keyword) have produced findings that suggest the nature of the effects of semantic elaboration depend upon whether the to-be-remembered word in question is a known word or a new word. Findings indicate that semantic elaboration does not facilitate new L1 word learning (Levin et al., 1982; Pressley, Levin, Kuiper, Bryant and Michener, 1982), including when it is operationalized via sentence writing conditions (Pressley, Levin and Miller, 1982). In rare instances, semantic elaboration has been found to be facilitative (e.g., Pressley, Levin, Kuiper, et al., 1982), but it can be argued that for the posttest measures used in these instances, the measures of lexical knowledge in question may not have been sensitive enough to one's degree of knowledge of new word form. Consistent with this interpretation, in their L1 word learning study, Pressley, Levin, Kuiper, et al. found that one semantic elaboration condition (imagery) was effective when the posttest was definition recall only, a test that provides word forms and does not require learners to generate words based on their own knowledge. However, the same researchers found another semantic elaboration condition (synonym) to be inhibitory (compared to no strategy) when the posttest was a matching task. Therefore, performance on 
the matching task depended more on knowledge of word form than did the recall task, because the matching task required selecting among multiple word forms at once, and therefore the inhibitory effect of semantic elaboration was observed.

This pattern of results is consistent with the transfer appropriate processing (TAP) theory (Morris et al., 1977) of human memory, which maintains that memorial effects depend upon the nature of tasks to be performed at study and test, and with the 'type of processing-resource allocation' (TOPRA) model for the relationship between semantic vs. structural processing and lexical learning (Barcroft, 2000). According to TAP, memory performance depends upon the extent to which processes engaged at the time of learning are the same as those engaged during a test of memory. According to the TOPRA model, when processing demands are sufficiently high (on limited processing capacity and task types, see Miller, 1956; Broadbent, 1958; Wickens, 1984), semantic elaboration can increase processing and learning of the semantic (conceptual) properties of words while simultaneously decreasing processing and learning of the structural properties of words (for evidence of a similar type of relationship at the sentence level, see VanPatten, 1990; 1996). The data indicating positive effects for semantic elaboration on measures less dependent on knowledge of word form and negative effects for semantic elaboration on measures more dependent on knowledge of word form (e.g., Pressley, Levin, Kuiper, et al., 1982) are consistent with both TAP theory and the TOPRA model for the following reasons. First, as predicted by TAP, they suggest that the memorial effect of semantic elaboration at study depends upon the nature of task to be performed at testing. Semantic elaboration at study may facilitate performance on tasks that depend more on memory for the semantic component of words (definition recall) but not tasks that depend more on memory for the formal component of words (a matching task). Secondly, as predicted by the TOPRA model, the data suggest that as semantically oriented processing and learning increase in a condition of semantic elaboration, semantically oriented learning increases but form-oriented processing and learning can decrease as a consequence, explaining the positive effect of 
semantic elaboration on a task such as definition recall and the negative effect of semantic elaboration on a task such as matching words and their definitions. Although the predictions of TAP and TOPRA are consistent at a general level, the TOPRA model focuses directly on the dissociation between different types of processing (e.g., semantic, structural) and the gradated relationship between types of processing and their learning counterparts.

McDaniel and Kearney (1984) and Barcroft (2002) provided additional support for this interpretation. McDaniel and Kearney found that semantic orienting conditions resulted in less vocabulary recall than an uninstructed condition, and Barcroft observed a double dissociation between semantic vs. structurally oriented elaboration and free recall of words in a known vs. new language (L2). In the latter study, free recall of words in a known language (English) was greater in the semantic elaboration condition (make pleasantness ratings about word referents), whereas free recall of words in the target L2 (Spanish) was greater in the structural elaboration condition (count letters in target words).

In an L2 lexical learning study, Brown and Perry (1991) found a Keyword-semantic condition to be more effective than Keyword alone but found no difference between a Keyword-semantic and a semantic only condition. Their results indicate that semantic elaboration itself (apart from a mnemonic device) did not have a positive effect on L2 word learning. In another L2 lexical study, Prince (1996) found translation-based learning to be more effective than sentence-level contextual learning, the latter of which one could associate with semantic elaboration, which again points towards a lack of effectiveness for semantic elaboration as new word learning technique.

Coomber et al. (1986) concluded positive effects for increased semantic elaboration on pseudoword learning in a study that compared the effects of three rehearsal methods (definition, examples, sentence composing) on performance on three types of posttests corresponding to the three learning conditions. In their study, sentence composing was deemed to involve the most semantic processing of the three methods and was found to be the most effective method overall. However, their conclusion regarding the positive 
effect of semantic elaboration may have been related to amounts of semantic elaboration that took place during each of their three methods. These amounts may have been different from the respective amounts hypothesized by the researchers. More specifically, for their definitions method, the participants had to process the new words based upon the words' semantic properties by comparing the meaning of each word to five different possible definitions and thereby make semantically oriented evaluations. This method actually could have involved greater amounts of semantic elaboration than their sentence writing condition.

The present study examined the effect of writing new words in sentences, a condition requiring semantic elaboration, during the initial stages of learning new L2 words. Participants in the no sentence writing condition of the study were not instructed to try to avoid thinking about word meaning altogether. In the sentence writing condition, however, the participants were required to elaborate on word meaning to the extent needed to be able to write each target word in an original sentence. Therefore, the study was designed to compare conditions associated with two levels of semantically oriented processing, as opposed to conditions with and without semantic processing. The dependent variable in the study was L2 vocabulary production, which required learners to demonstrate their degree of knowledge of the formal properties of words to a greater degree than would have been the case with other more receptively oriented measures that do not require learners to produce the target word forms (e.g., definition recall). In light of previous research, the effect of the semantic elaboration component of sentence writing was expected to have inhibitory effects on the participants' learning and subsequent ability to produce the words in question. This prediction is consistent with both TAP theory and with the TOPRA model's account of the relationship between semantic vs. structural processing and lexical learning.

\section{Output and new word learning}

An additional characteristic of writing new words in sentences is that it involves output. Whereas Krashen's Input Hypothesis 
(1985) focused on the critical role of input for second language acquisition, other researchers have also drawn attention to the role that output. Swain (1985) has argued that output may help learners by requiring them to move away from relying a great deal on top-down processing strategies and contextual cues used during comprehension towards relying more on specific means of expression and syntax needed to produce language. But what is the effect of output on L2 vocabulary acquisition? Does requiring learners to produce target L2 words positively affect L2 word learning?

Most studies on the role of output in L2 lexical acquisition have focused on the effects of different types of output activities during incidental vocabulary learning, that is, when learners are able to pick up new words from context (e.g., while reading for comprehension) without directly intending to do so. Overall, the results of these studies have been mixed in terms of the effects of output on lexical acquisition rates. For example, Watanabe (1997) found that having learners write the meaning of L2 words in their L1s while reading had no effect on lexical acquisition rates. Barcroft (1999), however, found that requiring learners to copy L2 words during word-picture repetition learning (output) decreased word form learning rates when compared to word-picture repetition learning alone (no output), suggesting that the processing resources required to produce the output may have reduced the amount of resources available to process the new words as input. On the other hand, Ellis and He (1999) found that learners in a modified output treatment achieved higher vocabulary acquisition scores than learners in two input groups (premodified input, interactionally modified input) after being exposed to a set of L2 words in a listenand-do task related to showing pieces of furniture in an apartment.

Overall, these findings on the relationship between different types of output and lexical learning suggest that the effect of output may depend heavily on the nature of the lexical learning task and the type of dependent measure in question. As such, the findings are consistent with TAP theory. The existing research also suggests that more research in this area is warranted. With regard to the present study, sentence writing conditions involving elicited 
output are compared to conditions without elicited output. Therefore, the results of the study should help to shed new light on how output may affect rates of new word learning as well. Whereas additional future research and analysis may be needed to tease apart the independent effects of the semantic elaboration and output components of sentence writing, the present study was designed to improve our understanding of how the task of sentence writing as a whole affects L2 lexical acquisition.

\section{Research questions}

This study addressed the following research questions:

1) Does writing new words in sentences affect L2 lexical acquisition?

2) If the answer to Question 1 is 'yes', does sentence writing decrease L2 lexical acquisition?

3) If the answer to Question 1 is 'yes', are the effects of sentence writing both short term and long term?

Two experiments were conducted to address these questions. Experiment 1 compared the effects of a sentence writing condition with a condition of repetition learning without sentence writing. Experiment 1 compared these two conditions in part to approximate real-world scenarios in which the tasks in question might really be used. Experiment 2, on the other hand, compared the effects of a sentence writing condition with a no sentence writing condition while holding constant number of repetitions and overall time on task in each condition. In so doing, Experiment 2 tested whether the results obtained in Experiment 1 could have been due to differences in number of repetitions or overall exposure time as opposed to being due to the sentence-writing vs. no sentence-writing conditions.

\section{Experiment 1}

1 Overview of the study

Experiment 1 compared the effects of writing new words in sentences (sentence writing) vs. word-picture repetition learning alone (no sentence writing) on new word learning rates among 


\section{Sentence writing in lexical acquisition}

second-semester L2 Spanish learners. The participants attempted to learn 24 concrete Spanish nouns while viewing each word along with its corresponding picture on a television screen. Each participant attempted to learn 12 words under the no sentence writing condition (repetition only) and 12 under the sentence writing condition. After the exposure phase, the participants completed three posttests: immediate, two days after learning and one week after learning. Posttests were scored, and scores were submitted to repeated measures analyses of variance that included condition and time as independent variables, and number of lexical items produced (score) as the dependent variable. ${ }^{1}$

\section{Participants}

The original pool of participants meeting the criteria for the study were 60 second-semester university-level L2 Spanish students enrolled in four different course sections at the University of Illinois at Urbana-Champaign. Of these 60 participants, only 45 participants appeared for all three posttests, 23 from two classes with one presentation order and 22 from two classes with the other presentation order. To obtain equal cells for counterbalancing, one participant was excluded at random among those who had first presentation order. Forty-four participants remained, 22 in each of the two orders. In addition to being pretested on the target words in the study, the participants met each of the following criteria: (1) the L1 of the participant was English; (2) the participant had no advanced knowledge of any other language besides English; and (3) Spanish was not spoken regularly in the participant's household.

\section{Design}

In this within-group experiment, each participant attempted to learn 12 words in the sentence writing condition and 12 words in

\footnotetext{
${ }^{1}$ The data and analyses presented here are a reduced version of those presented in Barcroft (2000), which included L2 experience and gender as independent variables, presentation order and class as blocking variables, and both raw and converted scores for number of lexical items produced as dependent variables.
} 
the no sentence writing condition. In order to counterbalance independent variables and presentation orders (word groups), half of the participants had Presentation Order 1 and the other half had Presentation Order 2. Participants 1-22 had Presentation Order 1; they wrote sentences while attempting to learn Words 1-12 and viewed 4 word-picture repetitions for each word without writing sentences while attempting to learn Words 13-24. Participants 23-44 had Presentation Order 2; they viewed 4 word-picture repetitions for each word while attempting to learn Words 1-12 and wrote sentences while attempting to learn Words $13-24$.

\section{Experimental words}

The 24 experimental words were selected according to three main criteria: (1) they were concrete nouns that could be represented visually in a non-ambiguous way; (2) there was a strong likelihood that the participants would not know the words; ${ }^{2}$ and (3) they were not easily recognizable cognates with English. In addition to counterbalancing word groups (presentation orders) with the independent variables, words of different lengths were included so as to reflect a range of real-word lexical items. A selection of two-, three-, four- and five-syllable words was included. In order to minimize differences in the relative difficulty of words in each of the two word groups, the average number of syllables and letters in each word group was (approximately) equalized. Table 1 presents the experimental words according to word group, number of syllables and number of letters. Note that $c h, l l$, and $r r$ also may be counted as single letters in Spanish, which would alter the letter counts in Table 1 to a minimal degree.

\footnotetext{
${ }^{2}$ In order to select 24 experimental words that the participants would most likely not already know, it was helpful that I had previously been an instructor of the second-semester Spanish course in which the participants were enrolled at University of Illinois at Urbana-Champaign. The same set of experimental words were also used in other experiments using third-semester Spanish students. It was therefore expected that second-semester Spanish students most likely would not know the words either. The use of the pretest as an independent check of knowledge of the target words was still critical, however. In addition, it would be beneficial for the participants to learn some or all of the experimental words used in the study (e.g., knowing the word for 'nail', 'shelf', or 'clothespin' can be useful).
} 
Table 1 Experimental words categorized according to word group, syllables and letters

Words $1-12$

\begin{tabular}{|c|c|c|c|c|c|}
\hline Word & Syllables & Letters & Word & Syllables & Letters \\
\hline 1. serrote & 3 & 7 & 13. aletas & 3 & 6 \\
\hline 2. regaderna & 4 & 8 & 14. resbaladilla & 5 & 12 \\
\hline 3. borla & 2 & 5 & 15. pala & 5 & 4 \\
\hline 4. rastrillo & 3 & 9 & 16. balde & 2 & 5 \\
\hline 5. embudo & 3 & 6 & 17. clavija & 3 & 7 \\
\hline 6. destornillador & 5 & 14 & 18. sacudidor & 4 & 9 \\
\hline 7. imán & 2 & 4 & 19. asa & 2 & 3 \\
\hline 8. clavo & 2 & 5 & 20. candado & 3 & 7 \\
\hline 9. taladro & 3 & 7 & 21. tenazas & 3 & 7 \\
\hline 10. cabestrillo & 4 & 11 & 22. estantería & 5 & 10 \\
\hline 11. pinza & 2 & 5 & 23. Iupa & 2 & 4 \\
\hline 12. chiringa & 3 & 8 & 24. gancho & 2 & 6 \\
\hline Total & 36 & 89 & Total & 36 & 80 \\
\hline Average & 3.0 & 7.4 & Average & 3.0 & 6.7 \\
\hline
\end{tabular}

\section{Exposure time and repetitions}

Differences in overall exposure time and number of repetitions between conditions were included in an effort to approximate realworld scenarios in which the tasks used in the study might take place. Overall exposure time was 24 seconds in the no sentence writing condition vs. 48 in the sentence writing condition. There were 4 repetitions of each word in the no sentence writing condition vs. 1 exposure to each word in the sentence writing condition. With regard to the sentence writing condition, it was decided that 24 seconds per word to complete a sentence writing task is a substantially limited amount of time and that L2 instructors in the real world might be inclined to allow a larger amount of time to complete this type of task. With regard to the no sentence writing condition, it was decided that (1) one long (24-second or 48 -second) repetition only of each word in the no sentence writing condition would have represented a less probable real-world example than 4 repetitions because the former would involve viewing single word-picture pairs for an extended period of time; and that (2) including more than 4 repetitions would have represented a less probable real-world example than only the 4 
repetitions because many more repetitions (e.g., 8 repetitions) would have become redundant and less likely to be reproduced in the real world in a similar fashion. Therefore, differences in exposure time and number of repetitions were included in consideration of the external validity of the study. In the no sentence writing condition, 4 presentations of each word were interspersed over the presentation sequence. Each time the words appeared in the same order. In the sentence writing condition, however, each word was presented only once. This difference in presentation pattern is discussed in greater detail in light of the finding that memory for spaced items are remembered better than massed items (the 'spacing effect', Melton, 1967) and in light of the results of Experiment 2 in the discussion section below.

\section{Procedure}

All data were collected in the participants' regular classrooms and during their regular class time. Each participant completed a language background questionnaire and was provided with general instructions for the experiment in written form. Each participant then completed the pretest on which the 24 experimental words appeared in Spanish in an order that was reverse to that in which they would appear during the learning and testing phases (in an attempt to avoid overfamiliarizing the participants with the exposure-phase ordering of the words during the pretest). On the pretest, all of the participants were asked to write the English word for any of the 24 Spanish words they knew, and to turn in the sheet when they had finished. None of the participants in the study correctly translated any of the 24 target words on the pretest.

After the pretest, all of the participants were instructed orally as follows:

In this experiment you'll be trying to learn new Spanish words by watching them appear on the television screen alongside of their pictures. There will be 24 words. For half of the words, you'll be writing each word in a sentence in Spanish. You'll have 48 seconds to write each sentence after the word and its picture appear on the television screen. For the other half of the words, you won't be writing sentences. Instead, you'll be seeing each of those 12 words four times each for 6 seconds each. Please do your best to learn all of the words. 
In the exposure phase, the participants were given a sheet with spaces for writing sentences and were instructed orally as to when and where to write sentences. For both conditions, the participants were instructed to do their best to learn the words. All of the participants were presented with word-picture pairs (numbered 1-24) on a television screen in the front of the classroom in timed intervals. The intervals were marked by beeps on a tape, and upon hearing each beep, the word-picture pairs were changed by hand on a projection device connected to the television. In the no sentence writing condition, word-picture pairs appeared 4 times successively on the screen for 6 seconds each. The time delay between learning Words 1-12 and learning Words 13-24 was approximately 30 seconds in every class.

Immediately after the exposure phase, the participants were given Posttest 1 and instructed orally as follows:

Please just do your best to remember and write the Spanish word for each picture you see. Each picture is numbered, so write the Spanish words in the space on your answer sheet next to that number. Write as much of each word as you can.

In Posttest 1, the same 24 pictures used in the exposure phase (numbered in the same way as 1-24) appeared in the same order, but this time without the Spanish words below them. Two days later, I returned at the beginning of class, and Posttest 2 was administered. Posttest 2 was exactly the same as Posttest 1 except that it was labelled 'Posttest 2' and that it was administered two days after learning. After Posttest 2, the participants were asked to answer the following question on the back of their answer sheets:

Since the last test you took as a part of this experiment, have you practiced or had any additional contact with the 24 Spanish words on the test? If 'yes,' please explain.

One week later, I returned to administer Posttest 3. Posttest 3 was exactly the same as Posttests 1 and 2, except that it was labelled 'Posttest 3' and that it was administered one week after the initial exposure phase. After Posttest 3, the participants were asked the same question about whether or not they practiced or had any additional contact with the 24 Spanish words between Posttests 2 and 3 . 
If any participant had written 'yes' to the question about additional exposure or practicing the words and indicated that they had practiced the target words beyond only 'thinking about them', their current posttest and (if taken) subsequent posttest would have been excluded from the data. However, none of the 44 participants in the experiment had to be excluded for this reason. The lowest number of sentences written by any one the 44 participants in the experiment was 6 . The average number of sentences written was 11.6 out of 12. Examples of sentences produced by the participants are shown in Appendix 1. As these examples suggest, participants were evaluating the semantic properties of the target words in order to write the sentences.

\section{Assessment}

The data were scored by independent evaluators based on (1) syllable scoring, or the number of syllables correctly produced for the target words in each condition (maximum $=36$ syllables in each condition), and (2) whole word scoring, or 1 point for each correctly produced word. 'Blind scoring' was used at all times. Using syllable scoring, the following scores would be assigned for the target word embudo 'funnel': 'emb...' $=1$, embu $=2$, embudo $=3$. For each participant, after scoring Words 1-24, scores for Words 1-12 and scores for Words 13-24 were totalled separately, providing a total score for the no sentence writing condition and a total score for sentence writing condition.

\section{Results}

a Analysis of results: Scores based on syllable scoring were subjected to a repeated measures analysis of variance (ANOVA) with condition and time as within-subject independent variables

${ }^{3}$ The larger data set reported in Barcroft (2000) were scored according to a different scoring protocol, LPSP-written, which allocates scores of $0, .25, .50, .75$ or 1 for each response based on percentages of letters produced or both produced and correctly placed within target words: $1=$ all letters correctly placed; $.75=50-99.9 \%$ correctly placed or $75-99.9 \%$ present; $.50=25-49.9 \%$ correctly placed or $50-74.9 \%$ present; $.25=$ any 1 letter correctly placed or $25-49.9 \%$ present; $0=$ all other responses. 
and score as the dependent variable. Scores based on whole word scoring were submitted to a second ANOVA with condition and time as independent variables and score as the dependent variable. The alpha level was set at .05 for all of the statistical analyses.

b Syllable scoring: The results based on syllable scoring indicated that the means for the no sentence writing condition were much higher than the means for the sentence writing condition. Table 2 displays means for condition over time in the top of the panel and overall means for condition in the bottom of the panel. Overall means were 9.64 for no sentence writing and 4.40 for sentence writing, a difference of $119 \%$. The results of the ANOVA revealed a significant main effect for condition, $F(1,43)=86.01$, $p<.001, \eta^{2}=.667$, a significant main effect for time, $F(2,86)=$ $40.56, p<.001, \eta^{2}=.485$, and no other significant main effects or interactions. The inhibitory effect of sentence writing maintained over time is displayed graphically in Figure 1.

$c$ Whole word scoring: The data analysed based on whole word scoring (1 point for each correctly produced word) yielded a similar pattern of results. Means (with standard deviations in parentheses) for no sentence writing were 2.91 (1.93) at Time 1, 2.61 (1.79) at Time 2, 2.73 (1.95) at Time 3 and 2.75 (1.80) overall. The lower means for sentence writing were 1.66 (1.51) at Time $1,1.16$ (1.26) at Time 2, 1.05 (1.29) at Time 3, and 1.29 (1.25) overall. Overall means were $113 \%$ higher for no sentence writing. The whole word

Table 2 Means based on syllable scoring for each condition in Experiment 1

\begin{tabular}{llrr}
\hline Time & Condition* & Mean & SD \\
\hline Immediate & No sentence writing & 11.61 & 6.13 \\
\multirow{2}{*}{ Two days later } & Sentence writing & 5.80 & 4.57 \\
& No sentence writing & 8.82 & 4.95 \\
One week later & Sentence writing & 3.70 & 3.94 \\
& No sentence writing & 8.48 & 5.30 \\
Overall & Sentence writing & 3.70 & 5.35 \\
& No sentence writing & 9.64 & 5.17 \\
& Sentence writing & 4.40 & 4.29 \\
\hline
\end{tabular}




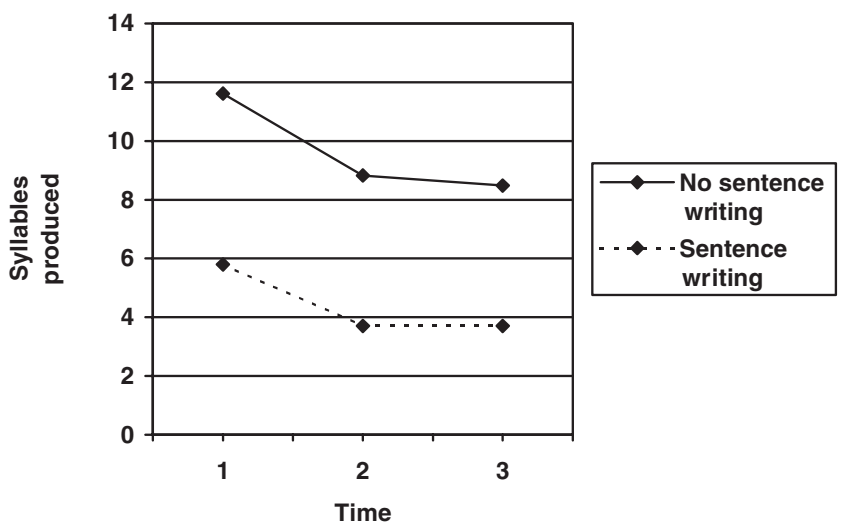

Figure 1 The effect of condition over time (Experiment 1)

scoring data were subjected to the same type of ANOVA used for syllable scoring. This ANOVA revealed significant main effects for condition, $F(1,43)=84.97, p<.001, \eta^{2}=.664$, and for time, $F(2,86)=9.88, \quad p<.001, \eta^{2}=.187$. No other significant main effects or interactions were observed.

\section{Discussion}

The results of Experiment 1 indicated that writing new words in sentences does affect L2 lexical acquisition based on performance on a productive measure of vocabulary knowledge. The results indicated that the effect was strongly negative and that the negative effect maintained over time. These results are consistent with the predictions of TAP theory in that positive effects observed for sentence writing in other learning paradigms for other types of dependent measures (e.g., definition recall) do not maintain for productively oriented L2 vocabulary learning. The results are also consistent with the predictions of the TOPRA model in that the increased semantic processing associated with writing words in sentences was found to have a negative effect on performance on a task that depended largely on form-oriented learning. The implications of these results are discussed further in Section V, after the presentation of Experiment 2, which addressed the issue of presen- 
tation intervals used in no sentence writing and sentence writing conditions further.

\section{Experiment 2}

In light of the strong negative effect observed for the sentence writing condition in Experiment 1, the purpose of Experiment 2 was to partially replicate Experiment 1 while controlling for number of repetitions and overall time on task in order to examine whether the effects observed for sentence writing were due to differences in presentation patterns in that study. The design, method and procedures used in Experiment 2 were similar to those used in Experiment 1. In Experiment 2, however, each target word was presented only once for 24 seconds each in both the no sentence writing and sentence writing conditions. In addition, the participants were asked to cover all previously written sentences in order to examine whether the negative effect of sentence writing observed in Experiment 1 may have been related to participants looking back at previously written sentences.

\section{Participants}

The original participant pool in Experiment 1 consisted of 22 English-speaking second-semester L2 Spanish students enrolled at Parkland University in Champaign, Illinois. The data provided by 8 participants were excluded based on task performance issues (not writing sentences as instructed, writing down words instead of sentences in the no sentence writing conditions or looking back at previously written sentences). Of the remaining 14 participants, 6 were from one section of the Spanish course and 8 were from another section. Four of these participants were excluded because they were not present for the delayed posttest, leaving 10 remaining participants. These participants met the same criteria as in Experiment 1.

\section{Design}

The basic design of Experiment 2 was the same as in Experiment 1. 


\section{Experimental materials}

The experimental materials used in Experiment 2 were similar to those used in Experiment 1, except that the 24 flash cards with the target words were presented directly to the students by the investigator at the front of the class because the same presentation device was not available at the university in question. A watch was also used to control for time. Finally, experimental packets were provided for each participant with general instructions on the first page and instructions for the no sentence writing and sentence writing tasks (with 12 numbered boxes for writing sentences) on the following two pages; two posttests, with 24 numbered spaces on which to write the target Spanish words; and a questionnaire about strategy use, self-assessment of performance, and other comments related to the experiment.

\section{Experimental words}

The 24 experimental words were the same as those used in Experiment 1 .

\section{Procedure}

Data were collected according to similar procedures as in Experiment 1. In the learning phase of Experiment 2, however, the participants were instructed to turn the pages of their experiment booklets when instructed to do so and to follow the instructions on each page. Within each class, half of the students were given 'no sentence writing' instructions (on the first page of the booklet) for the first 12 words and 'sentence writing' instructions for the second 12 words (on the second page of the booklet) (Presentation Order 1). The other half of the class was given the reverse (Presentation Order 2). In contrast to Experiment 1, each word in each group of 12 words was presented only once for 24 seconds at the front of the class. Finally, Posttests 1 and 2 were administered as in Experiment 1. Only one delayed posttest, Posttest 2, was administered, however. The larger drop in overall scores in Experiment 1 occurred between Posttests 1 and 2 and not between Posttests 2 
and 3, making inclusion of the time delay between Posttests 1 (immediate) and 2 (2 days later) particularly important when examining for a possible time by condition interaction. The lowest number of sentences written by any of the 10 participants was 7 . The average number of sentences written was 10.7 out of 12 .

\section{Results}

a Assessment and analysis of results: Assessment and analyses of results were the same as in Experiment 1 except that only two levels of time (immediate, 2 days later) were analysed.

\section{$b$ Syllable scoring: The results of Experiment 2 based on syllable} scoring indicated that the means for the no sentence writing condition were much higher than the means for the sentence writing condition. Table 3 displays means for condition over time in the top of the panel and overall means for condition in the bottom of the panel. Overall means were 10.30 for no sentence writing and 4.95 for sentence writing, a difference of $108 \%$. The results of the ANOVA revealed a significant main effect for condition, $F(1,9)=12.18, p=.007, \eta^{2}=.575$, and no other significant main effects or interactions. The effect of time did not reach a level of statistical significance, $F(1,9)=4.13, p=.073, \eta^{2}=.315$. The negative effect of sentence writing is displayed graphically in Figure 2.

c Whole word scoring: Based on whole word scoring, means (with standard deviations in parentheses) for the no sentence writing condition were 2.50 (2.46) at Time 1, $1.70(1.49)$ at Time 2 and 2.10 (1.82) overall, as compared to the lower means for the

\footnotetext{
${ }^{4}$ The data from Experiment 2 were also scored based on LPSP-written scoring (Barcroft, 2000) with results similar to those based on syllable scoring. Based on LPSP-scoring, means (with standard deviations in parentheses) for no sentence writing were 5.55 (3.25) at Time 1, 4.70 (2.73) at Time 2 and 5.13 (2.94) overall. The lower means for sentence writing were 3.05 (3.15) at Time 1, 2.28 (2.63) at Time 2 and 2.66 (2.88) overall. The data based on LPSP-written scoring were subjected to the same type of repeated measures ANOVA used for syllable scoring. This ANOVA revealed a significant main effect for condition, $F(1,9)=13.10$, $p=.006, \eta^{2}=.593$, and a significant main effect for time, $F(1,9)=8.79, p=.016$, eta ${ }^{2}=.494$.

No other significant main effects or interactions were observed.
} 
Table 3 Means based on syllable scoring for each condition in Experiment 2

\begin{tabular}{llrr}
\hline Time & Condition* & Mean & SD \\
\hline Immediate & No sentence writing & 11.60 & 9.45 \\
& Sentence writing & 5.50 & 7.47 \\
Two days later & No sentence writing & 9.00 & 7.30 \\
& Sentence writing & 4.40 & 5.64 \\
Overall & No sentence writing & 10.30 & 8.18 \\
& Sentence writing & 4.95 & 6.53 \\
\hline
\end{tabular}

Note: ${ }^{*} n=10$ in each condition

sentence writing condition, which were 1.60 (2.22) at Time $1,1.40$ (1.84) at Time 2 and 1.50 (2.03) overall. The results of the ANOVA based on whole word scoring revealed no significant main effects or interactions. This lack of significant effect based on whole word scoring in Experiment 2 may be attributable to the lack of precision of whole word scoring as a measure of degree of word form knowledge in combination with the smaller sample in Experiment 2 as compared to Experiment 1.

\section{Discussion}

The results of Experiment 2 also indicated that writing new L2 words in sentences negatively affected L2 lexical learning for those words based on the productively oriented measure of L2 vocabulary knowledge examined. These results are also consistent with the predictions of TAP theory and the TOPRA model for the

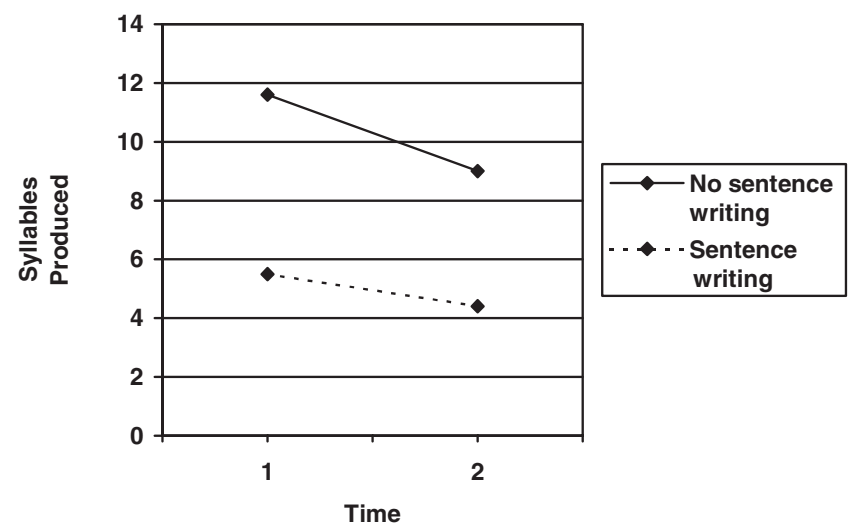

Figure 2 The effect of condition over time (Experiment 2) 
same reasons previously described for Experiment 1. In addition, the results of Experiment 2 based on syllable scoring indicated that negative effects observed for sentence writing in Experiment 1 cannot be accounted for based on differences in the presentation format used in Experiment $1 .{ }^{5}$ In Experiment 2, the effects of sentence writing based on syllable scoring remained strongly negative when a single 24-second repetition presentation format was used in both conditions. Furthermore, because Experiment 2 included controls to show that the participants had not looked back at their previously written sentences, the results of Experiment 2 also suggest that the negative effects observed for sentence writing in Experiment 1 cannot be accounted for on the basis that participants might have looked back at previously written sentences in the sentence writing condition and somehow confused target words and their meanings. Therefore, the results of Experiment 2 corroborate and extend the findings of Experiment 1.

\section{General discussion}

The results of both experiments revealed that writing new words in sentences negatively affected performance on a productively oriented measure of L2 lexical acquisition when compared to word-picture repetition learning alone (Experiment 1) and when compared to a no sentence writing condition using the same presentation format (Experiment 2). The negative effect of sentence writing based on syllable scoring also maintained over time in both experiments. The main theoretical implications of the study concern the relationship between semantic elaboration, output and L2 word-level input processing. These implications are discussed in the next three sections.

\footnotetext{
${ }^{5}$ Even if the observed effect had been attributable to additional repetitions in the no sentence writing condition, the finding would still be interesting from a theoretical and pedagogical standpoint for the following reasons. First, it would have been difficult to have predicted the observed effect (and its clear nature) beforehand without actually comparing the two conditions empirically. Secondly, effective vocabulary instruction is a reflection of how much one learns (develops) divided by the amount of time one invests. Therefore, even though one instructional technique (e.g., word-picture pair repetition learning) by nature may involve more repetition than another (e.g., writing new words in sentence), the relative effectiveness of the techniques can still be examined and evaluated by considering gain divided by time.
} 


\section{Semantic elaboration as a double-edged sword}

The finding that sentence writing can have a strong inhibitory effect on new word learning is consistent with the TAP theory of human memory and with the TOPRA model for L2 lexical learning: Although semantic elaboration can facilitate memory for known words, it may not facilitate and can even inhibit memory for new word forms. The results of the present study emphasize the importance of distinguishing between learning that is semantic in nature (semantic learning) and learning that is directed towards the formal properties of stimuli (form learning) when predicting the potential effects of semantic elaboration on learning. In short, semantic elaboration can function like a double-edged sword during word-level input processing: When processing resources are being utilized at maximum levels, increased semantic processing can facilitate semantic learning while concurrently inhibiting form processing and form learning (Barcroft, 2000; 2002). Consistent with this account, learning the target words in the no sentence writing condition resulted in better performance on the measures of productive knowledge because in this condition the participants were allowed to focus more on the formal properties of the target words and because the posttest task measured the participants' knowledge of the formal properties of the target words to a sufficient degree. Upon further consideration of the predictions of TAP, one might also predict that the sentence writing condition could produce better performance on an alternative type of dependent measure, such as a posttest that depended less on knowledge of the formal properties of the target words and more on other aspects. Furthermore, the theoretical account described and the results of the study in no way deny that access to word meaning is necessary for successful word learning nor that focus on word meaning can be critical; they simply point to how the multiple subcomponents of word knowledge (e.g., form, meaning, mapping) are dissociable and differentially facilitated or impeded by different types of cognitive processing.

Could the effects observed in Experiment 1 of this study have been due solely to the increased number of repetitions in the no sentence writing condition as compared to the sentence writing 
condition and not to the inhibitory effects of semantic elaboration? Studies have found that memory for items presented in an interspersed manner (spaced items) are remembered better than items presented massed together (massed items): the 'spacing effect' (Melton, 1967). It could therefore be argued that the 'massed' presentation pattern used for the sentence writing condition (one long repetition for 48 seconds) vs. the 4 interspersed repetitions (for 6 seconds each) in the no sentence writing condition might have been responsible for yielding the observed effects in Experiment 1. The results of Experiment 2 indicated, however, that the negative effect of sentence writing was not dependent on having more repetitions or interspersed target words in the no sentence writing condition.

The inhibitory effect observed for sentence writing is consistent with previous studies showing (1) inhibitory effects for semantic elaboration on new word learning (e.g., Pressley, Levin and Miller, 1982: Experiment 4), (2) variant effects of semantic elaboration depending on the nature of the learning task in question, or transfer appropriate processing (McDaniel and Kearney, 1984; Morris et al., 1977), and (3) a double dissociation between semantic vs. structural orientation and free recall of known vs. new words (Barcroft, 2002). The results of this study are not consistent, however, with Coomber et al.'s finding that sentence composing had a positive effect. However, one may consider how semantic elaboration was operationalized in Coomber et al.'s study as a means of accounting for both sets of results. Although the sentence composing method in Coomber et al.'s study was deemed to involve more semantic processing than the other two methods examined, the amount of semantic processing required by the other methods actually might have been greater. As mentioned previously, their definitions method, which resulted in lower performance than the sentence composing task, required participants to compare each word's meaning with five different possible definitions. This task clearly involves a substantial amount of semantic elaboration (in order to compare appropriate vs. inappropriate meanings), possibly even more semantic elaboration than the sentence composing condition. It therefore may have been that the relatively greater amount of semantic elaboration in 
the definitions condition resulted in worse vocabulary learning performance than in the sentence composing condition.

\section{Inhibitory effects for output?}

The finding of an inhibitory effect for sentence writing on new word learning also suggests a potentially inhibitory role for output on some aspects of L2 lexical learning. Previous studies have found null effects (Watanabe, 1997), negative effects (Barcroft, 1999) and positive effects (Ellis and He, 1999) for output during vocabulary learning. Given that output is required to write new words in sentences, the results of the present study are most consistent with those of Barcroft (1999), who found that copying down new words (as compared to no word writing) significantly decreased new word learning rates, based on a measure of productive lexical knowledge. It is therefore likely that the large inhibitory effect observed for sentence writing in the present study was in part due to the elicited output component of sentence writing. However, comparison of the effects observed for the word copying condition as compared to the sentence writing condition in the present study help to shed further light on this issue. An overall negative effect of approximately $33 \%$ has been observed for copying words within an similar experimental paradigm to that of the present study (based on data reported by Barcroft, 1999), whereas the overall negative effect observed for sentence writing was approximately $119 \%$ in Experiment 1 and 108\% in Experiment 2 of the present study. This comparison suggests that the entire negative effect observed for sentence writing may not be explained as the result of writing down target words themselves. A future study comparing the effects of writing target words in selfgenerated sentences vs. copying entire sentences that contain target words could help to isolate further the effects of the semantic elaboration vs. output (writing in general) components of sentence writing. If one predicts that semantic elaboration should produce negative effects (i.e., predictions of TOPRA), one would expect the self-generated condition to produce greater negative effects than the sentence copying condition, given appropriate controls for other factors, such as sentence length. 
To what extent, however, was the strong inhibitory effect observed in this study due to the required use of L2 grammar and L2 lexical items (in the sentence writing condition) as compared to the involvement of increased semantic processing or the involvement of elicited output in general terms? The results of another experiment reported by Barcroft (1999) also suggested that the particular challenges associated with writing in L2 did not account for the negative effects of sentence writing observed in the present study. In that experiment, English-speaking L2 learners of Spanish attempted to learn 12 words by writing them in sentences in Spanish and 12 words by writing them in sentences in English and inserting Spanish words where the corresponding English words should appear. Although the means were higher in the English sentence writing condition, no significant differences were observed. This finding suggests that the particular challenges involved in writing in an L2 (e.g., as opposed to writing in an L1) were not the primary cause of the effects observed in the present study. Therefore, the potentially inhibitory effects of increased semantic processing and of any form of elicited output of any form become more viable candidates to implicate in order to account for the observed effects.

As with the hypothesized double-edged effect for semantic elaboration, the proposition that output can inhibit new word learning is consistent with TAP theory, the TOPRA model and the general finding that divided attention during time-sharing activities can inhibit learner performance given learners' limited processing capacities (Wickens, 1984). Although output may be necessary or useful for the development of certain components of L2 competence (Swain, 1985), it can potentially inhibit other areas of linguistic development, such as learners' ability to encode and retain new word forms when they initially encounter new L2 words.

\section{Summary of implications for word-level input processing}

As previously mentioned, research on word-level input processing (how learners process individual L2 words as input) is interested in how learners allocate processing resources to different aspects of new word learning (e.g., word form, form-meaning mapping). 
Combined with the results of previous studies, the present results suggest that both the increased semantic elaboration and forced output associated with sentence writing can inhibit learners' ability to encode new word form when overall processing demands are sufficiently high. With regard to semantic elaboration, the present results are consistent with the predictions of TAP theory and the TOPRA model with regard to word-level input processing: Although increased semantic processing can increase learning rates for the semantic properties of words, it can simultaneously decrease learning rates for the formal properties of new words by drawing resources away from word form processing. With regard to elicited output, the combined results of previous studies and the present study suggest that different subtasks associated with output, such as grammatical and lexical processing and the motor activity required for writing, can also exhaust processing resources that could otherwise be utilized to encode new word form during word-level input processing. Therefore, because both the semantic elaboration and elicited output components of sentence writing utilize processing resources that could otherwise be used to encode the new word form being presented as input, the strong inhibitory effects observed for sentence writing in the present study make sense.

\section{Directions for future research}

Although the findings of this study provide evidence that increased semantic processing or output may impede learning new word forms, there is a need to isolate the effects of these variables independently. Previous studies have found null effects (e.g., Levin et al., 1982) and inhibitory effects (e.g., Pressley, Levin, Kuiper et al., 1982) for semantic elaboration as well as null effects for output (Watanabe, 1997) and inhibitory effects for output via copying words (Barcroft, 1999) on new word learning. The present finding of an inhibitory effect for sentence writing is consistent with all of these. However, it would be useful for future studies to continue to investigate the independent effects of semantic elaboration and output on new word learning. One method of examining the effects of semantic elaboration on new word learning is to 
conduct partial replication studies of previous 'levels-of-processing studies' on semantic vs. structural elaboration while using new words (e.g., new L2 words) as opposed to known words (e.g., L1 words) as the to-be-remembered stimuli. To advance our understanding of the effects of output, on the other hand, future studies could compare the effects of activities that require learners to produce different amounts of output within the same time frame. Additionally, more work on the effects of both spoken and written output is warranted.

It also may be useful for more future lexical acquisition studies to employ posttests that are more sensitive to one's degree of knowledge of the formal component of new word learning. Insufficient measurement of the formal component of new word learning may be one reason why more null or inhibitory effects for semantic elaboration on new word learning have not been found more frequently in previous studies. The dependent variable in studies on semantic processing and lexical learning has often been more receptively oriented knowledge (e.g., definition recall) as opposed to more productively oriented knowledge (e.g., word production). The inhibitory effects found for sentence writing in this study may have been due to the use of posttest measures that are more sensitive to one's productive knowledge. When new word forms are provided for the learners, as is the case with measures of receptive knowledge, learners must exhibit their ability to connect word forms with their referents, but they do not have to generate word forms on their own. However, because lexical production does require learners to generate word forms on their own, lexical production may be a more precise measure of one's knowledge of the formal component of new word learning and thereby critical in examining the effects of a given variable on this particular component.

Finally, future studies may also address other issues related to generalizability. For example, one could examine different variations on the tasks involved in the present study, such as different L1s and L2s and different L2 proficiency levels. The proficiency level of the participants in the present study was relatively low, as indicated by many of the L2 sentences they generated (examples in 
Appendix 1). It is possible that the nature of the effect of sentence writing might be different among learners at higher L2 proficiency levels. Maintenance of other methodological aspects of the present study, such as its within-group design, use of time controls, counterbalancing of word groups, and use of a scoring protocol sensitive to partial word production, may be useful in future studies on these issues.

\section{Acknowledgements}

The first experiment of the study presented in this article was conducted for my doctoral dissertation, which was completed at University of Illinois at Urbana-Champaign in 2000. I would like to thank Bill VanPatten, Molly Mack, Diane Musumeci, and Anna Maria Escobar for their help with this project. I also would like to extend my gratitude to three anonymous Second Language Research reviewers for their helpful suggestions regarding an earlier version of this article.

\section{References}

Atkinson, R.C. and Raugh, M.R. 1975: An application of the mnemonic keyword method to the acquisition of a Russian vocabulary. Journal of Experimental Psychology: Human Learning and Memory 104, 126-33.

Barcroft, J. 1999: Processing resources and L2 lexical acquisition in three writing tasks. Paper presented at the Second Language Research Forum, Minneapolis, MN.

— 2000: The effects of sentence writing as semantic elaboration on the allocation of processing resources and second language lexical acquisition. Unpublished doctoral dissertation, University of Illinois at Urbana-Champaign, Urbana, IL.

- 2002: Semantic and structural elaboration in L2 lexical acquisition. Language Learning 52, 2.

Bower, G.H. and Reitman, J.S. 1972: Mnemonic elaboration in multilist learning. Journal of verbal learning and verbal behavior 11, $478-85$.

Broadbent, D.E. 1958: Perception and communication. New York: Pergamon. 
Brown, T. and Perry, Jr., F. 1991: A comparison of three learning strategies for ESL vocabulary acquisition. TESOL Quarterly 25, 655-70.

Cohen, A.D. 1987: The use of verbal and imagery mnemonics in secondlanguage vocabulary learning. Studies in Second Language Acquisition 9, 43-62.

Coomber, J.E., Ramstad, D.A. and Sheets, D.R. 1986: Elaboration in vocabulary learning: a comparison of three rehearsal methods. Research in the Teaching of English 20, 281-93.

Craik, F.I.M. and Lockhart, R.S. 1972: Levels of processing: a framework for memory research. Journal of Verbal Learning and Verbal Behavior 11, 671-84.

Craik, F.I.M. and Tulving, E. 1975: Depth of processing and the retention of words in episodic memory research. Journal of Experimental Psychology: General 104, 268-94.

Ellis, R. and He, X. 1999: The role of modified input and output in the incidental acquisition of word meanings. Studies in Second Language Acquisition 21, 285-301.

Epstein, M.L., Phillips, W.D. and Johnson, S.J. 1975: Recall of related and unrelated word pairs as a function of processing level. Journal of Experimental Psychology: Human Learning and Memory 104, 149-52.

Hyde, T.S. and Jenkins, J.J. 1969: The differential effects of incidental tasks on the organization of recall of a list of highly associated words. Journal of Experimental Psychology 82, 472-81.

Johnson-Laird, P.N., Gibbs, G. and de Mowbray, J. 1978: Meaning, amount of processing, and memory for words. Memory and Cognition 6, 372-75.

Johnson, D.D. and Pearson, P.D. 1978: Teaching reading vocabulary. New York: Holt, Rinehart, and Winston.

— 1984: Teaching reading vocabulary. 2nd edition. New York: Holt, Rinehart and Winston.

Krashen, S. 1985: The input hypothesis: issues and implications. New York: Longman.

Laufer, B. 1997: Incidental vocabulary acquisition: in praise of output. Paper presented at the Second Language Research Forum, East Lansing, MI.

Levin, J.R., McCormick, C.B., Miller, G.E., Berry, J.K. and Pressley, M. 1982: Mnemonic vs. nonmnemonic vocabulary-learning strategies for children. American Educational Research Journal 19, 121-36.

McDaniel, M.A. 1984: the role of elaborative and schema processing in story memory. Memory and Cognition 12, 46-51.

McDaniel, M.A and Kearney, E.M. 1984: Optimal learning strategies and their spontaneous use: the importance of task-appropriate processing. Memory and Cognition 12, 361-73. 
Melton, A.W. 1967: Repetition and retrieval from memory. Science 158, 532.

Miller, G. 1956: The magical number seven, plus or minus two: some limits on our capacity for processing information. Psychological Review 63, 81-97.

Morris, C.D., Bransford, J.D. and Franks, J.J. 1977: Levels of processing vs. transfer appropriate processing. Journal of verbal learning and verbal behavior 16, 519-33.

Pressley, M., Levin, J.R., Kuiper, N.A., Bryant, S.L. and Michener, S. 1982: Mnemonic vs. non-mnemonic vocabulary-learning strategies: additional comparisons. Journal of Educational Psychology 74, 693-707.

Pressley, M., Levin, J.R. and Miller, G.E. 1982: The keyword method compared to alternative vocabulary-learning strategies. Contemporary Educational Psychology 7, 50-60.

Prince, P. 1996: Second language vocabulary learning: the role of context vs. translations as a function of proficiency. The Modern Language Journal 80, 478-93.

Ross, B.H. 1981: The more, the better? Number of decisions as a determinant of memorability. Memory and Cognition 9, 23-33.

Schulman, A.I. 1974: Memory for words recently classified. Memory and Cognition 2, 47-52.

Stahl, S. and Fairbanks, M. 1986: The effects of vocabulary instruction: a model-based meta-analysis. Review of Educational Research 56, $72-110$.

Stevenson, R.J. 1981: Depth of comprehension, effective elaboration, and memory for sentences. Memory and Cognition 9, 169-76.

Swain, M. 1985: Communicative competence: some roles of comprehensible input and comprehensible output in its development. In Gass, $\mathrm{S}$. and Madden, C., editors, Input in second language acquisition. Rowley, MA: Newbury House, 235-52.

Tresselt, M.E. and Mayzner, M.S. 1960: A study of incidental learning. Journal of Psychology 50, 339-47.

VanPatten, B. 1990: Attending to content and form in the input: an experiment in consciousness. Studies in Second Language Acquisition 12, 287-301.

- 1996: Input processing and grammar instruction: theory and research. Norwood, NJ: Ablex.

Watanabe, Y. 1997: Input, intake and retention: effects of increased processing on incidental learning of foreign language vocabulary. Studies in Second Language Acquisition 19, 287-307.

Wickens, C.D. 1984: Processing resources in attention. In Parasuramanand, R. and Davies, D., editors, Varieties of attention. New York: Academic Press, 63-102. 
Appendix 1 Examples of sentences produced by participants

\section{Participant 1}

Yo nado con unas aletas.

'I swim with fins.'

Yo tengo un candado para mi bicicleta. 'I have a lock for my

bicycle.'

* Me gusta jugar en el resbaladilla. 'I like to play on the slide.'

* La clavija es malo para los niños. 'The plug is bad for children.'

\section{Participant 23}

*Una serrote usa para trabaja. 'A saw is used for working.'

* La borla es en el capa. 'The tassel is on the cap.'

*Imán attracta las metallicos. 'Magnet glass attracts metallic items.'

Chiringas son para la primavera. 'Kites are for the spring.' ( ${ }^{*}$ denotes sentences that contain grammatical or lexical errors.) 